8-1-1990

\title{
Random-Field Blume-Capel Model: Mean-Field Theory
}

\author{
Miron Kaufman \\ Cleveland State University, m.kaufman@csuohio.edu \\ M. Kanner
}

Follow this and additional works at: https://engagedscholarship.csuohio.edu/sciphysics_facpub

Part of the Physics Commons

How does access to this work benefit you? Let us know!

\section{Publisher's Statement}

Copyright 1990 American Physical Society. Available on publisher's site at http://link.aps.org/ doi/10.1103/PhysRevB.42.2378.

\section{Original Citation}

Kaufman, Miron and M. Kanner. "Random-Field Blume-Capel Model: Mean-Field Theory." Physical Review B 42 (1990): 2378-2382.

\section{Repository Citation}

Kaufman, Miron and Kanner, M., "Random-Field Blume-Capel Model: Mean-Field Theory" (1990). Physics Faculty Publications. 16.

https://engagedscholarship.csuohio.edu/sciphysics_facpub/16

This Article is brought to you for free and open access by the Physics Department at EngagedScholarship@CSU. It has been accepted for inclusion in Physics Faculty Publications by an authorized administrator of EngagedScholarship@CSU. For more information, please contact library.es@csuohio.edu. 


\title{
Random-field Blume-Capel model: Mean-field theory
}

\author{
Miron Kaufman and Michael Kanner \\ Department of Physics, Cleveland State University, Cleveland, Ohio 44115
}

(Received 27 February 1990)

\begin{abstract}
The global phase diagram of the Blume-Capel model in a random field obeying the bimodal symmetric distribution is determined by using the mean-field method. The phase diagram includes an isolated ordered critical end point and two lines of tricritical points. A new phase emerges for strong enough random fields: the ferromagnetic-nonmagnetic phase. It is argued that such a phase occurs in three dimensions.
\end{abstract}

\section{INTRODUCTION}

Quenched randomness causes first-order transitions to be replaced by continuous transitions in two dimensions, and the tricritical temperature to be lowered in higher dimensions. This was recently shown by Hui and Berker ${ }^{1}$ and Aizenman and $W e h r^{2}$ by using respectively positionspace renormalization-group techniques and rigorous methods. It is our goal in this paper to further study the effect of random fields on a tricritical phase diagram by employing the mean-field approximation, which is a reasonable approximation for high dimensions, and it is exact $^{3}$ for the equivalent-neighbor lattice.

The simplest spin model exhibiting a tricritical phase diagram in the absence of randomness is the Blume-Capel model. ${ }^{4}$ This model and its generalizations were used to simulate the thermodynamics of a variety of systems such as $\mathrm{UO}_{2}$ and ${ }^{3} \mathrm{He}-{ }^{4} \mathrm{He}$ mixtures, ${ }^{5}$ and it has been studied extensively with a variety of techniques: mean-field, ${ }^{4,5}$ position-space renormalization group,, 6 and other methods. ${ }^{8}$ In this paper we study the Blume-Capel model in the presence of a random magnetic field, which takes two values $\pm H$ with equal probability.

The Blume-Capel model includes two thermodynamic fields: the temperature $T$ and the crystal field $D$ conjugated to $-s^{2}$, where $s$ is the spin, which can take three values, \pm 1 and 0 . At zero temperature there are two phases: the ferromagnetic phase $(m \equiv\langle s\rangle= \pm 1$, $\left.Q \equiv\left\langle s^{2}\right\rangle=1\right)$ for small $D$, and the nonmagnetic phase ( $m=0, Q=0$ ) for large $D$. These phases coexist at some intermediate value of $D$. When the bimodal random field $\pm H$ is turned on, a novel phase emerges: the mixed ferromagnetic-nonmagnetic phase, $m= \pm \frac{1}{2}, Q=\frac{1}{2}$. In the plane $H, D$, for intermediate values of $H$, this phase occupies a buffer separating the ferromagnetic and nonmagnetic phases. Thus the emergence of the mixed phase can be viewed as a new manifestation of the weakening of the first-order transitions by the randomness: the jump in $m$ from \pm 1 to 0 is replaced by two smaller jumps: \pm 1 to $\pm \frac{1}{2}$ and $\pm \frac{1}{2}$ to 0 .

We believe that the emergence of the mixed phase is not a mere artifact of the mean-field approximation, and should also occur in three dimensions. At low temperatures and large $D$ the nonmagnetic phase ( $m=0, Q=0)$ is stable, while at low temperatures and large $H$ the paramagnetic phase $(m=0, Q=1)$ is stable. A direct transition between these planes, for large values of both $H$ and $D$, is unlikely ${ }^{9}$ because it will involve a discontinuity in $Q$ but no discontinuity in $m$. A continuous transition ( $Q$ decreases from unity to zero in the paramagnetic phase) could hold in two dimensions. In three dimensions however, we expect the emergence of the new phase between the paramagnetic and nonmagnetic phases to allow for jumps in both $m$ and $Q$ at the two phase boundaries.

The mixed ferromagnetic-nonmagnetic phase persists for arbitrarily large $H$ provided the crystal field $D$ is also large. The existence of long-range order $(|m|>0)$ for unbounded values of the random-field strength is also observed in the Ising model in a trimodal random-field, ${ }^{10}$ but not for the bimodal ${ }^{11}$ and Gaussian ${ }^{3}$ distributions.

We find in the three-dimensional parameter space $T, H, D$ that there are two noncontiguous tricritical lines. The first starts at the pure Blume-Capel tricritical point ${ }^{4,5}$ and shows a monotonic decrease of the tricritical temperature when $H$ is increased from zero. This is a manifestation of the weakening of first-order transitions by random fields. ${ }^{1}$ The second line which starts at the Aharony tricritical point of the Ising model in a binary random field, $D=-\infty$, does not show this simple behavior but exhibits an extremum corresponding to a double tricritical point. Thus moderate to large randomness has a subtler and more complicated effect than weak randomness on the phase diagram. In the same context we note the occurrence of reentrance phenomena and of nonmonotonic dependence of the densities $m$ and $Q$ on the thermodynamic fields.

Besides the tricritical points the global phase diagram also includes the following multicritical points: a line of ordered critical points ${ }^{10}$ (two coexisting critical phases) and an isolated ordered critical end point (coexistence of two critical phases and a disordered phase).

The remainder of this article contains the solution of the Blume-Capel model with a bimodal random field in Sec. II, and our conclusions in Sec. III.

\section{MODEL AND SOLUTION}

At each site of the equivalent-neighbor lattice (the mean-field model) there is a spin $s_{i}= \pm 1,0$. The Hamil- 
tonian associated with the $N$ spins is

$$
-\mathcal{H} / T=\frac{J}{2 N} \sum_{i, j} s_{i} s_{j}-J D \sum_{i} s_{i}^{2}+J \sum_{i} H_{i} s_{i},
$$

where $J \equiv 1 / T$. The magnetic field $H_{i}$ is distributed according to the bimodal distribution:

$$
P\left(H_{i}\right)=\frac{1}{2}\left[\delta\left(H_{i}-H\right)+\delta\left(H_{i}+H\right)\right],
$$

where $H \geq 0$ measures the strength of the random field and $\delta$ is the Dirac $\delta$ function.

In the thermodynamic limit the quenched averaged free energy per spin is ${ }^{3}$

$$
f=\frac{1}{J} \min _{m} \Phi(m)
$$

where

$$
\Phi=\frac{1}{2} J m^{2}-\left\langle\ln \left\{1+e^{-J D} 2 \cosh \left[J\left(m+H_{i}\right)\right]\right\}\right\rangle .
$$

The quenched average $\langle\cdots\rangle$ in Eq. (4) is performed by using the distribution in Eq. (2). The value of $m$ which minimizes $\Phi$ is the average site magnetization $\left\langle s_{i}\right\rangle$ while the average of $s_{i}^{2}$ is

$$
Q=\frac{\delta f}{\delta D}=\left\langle\frac{e^{-D J} 2 \cosh \left[J\left(m+H_{i}\right)\right]}{1+e^{-D J} 2 \cosh \left[J\left(m+H_{i}\right)\right]}\right\rangle .
$$

\section{A. Zero temperature}

The ground-state energies determine the phase diagram at zero temperature or $J=\infty$. We find four phases:

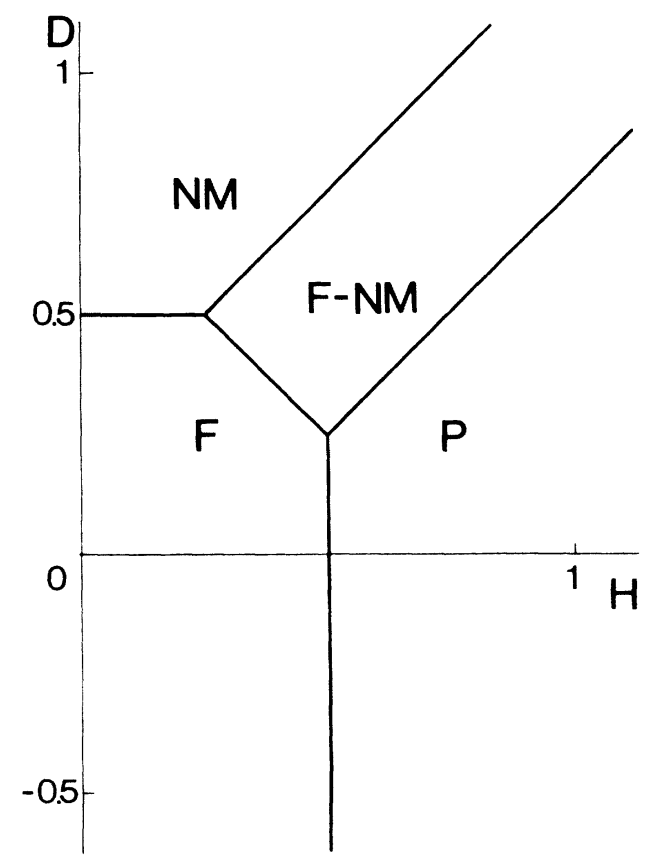

FIG. 1. The zero-temperature phase diagram. The four phases: ferromagnetic (F), nonmagnetic (NM), paramagnetic (P), and ferromagnetic-nonmagnetic (F-NM) are separated by lines of first-order transitions. the ferromagnetic phase ( $m= \pm 1, Q=1, f=D-\frac{1}{2}$ ); the nonmagnetic phase $(m=0, Q=0, f=0)$; the paramagnetic phase $(m=0, \quad Q=1, \quad f=D-H)$; the ferromagnetic-nonmagnetic phase $\left[m= \pm \frac{1}{2}, \quad Q=\frac{1}{2}\right.$, $\left.f=\frac{1}{2}\left(D-H-\frac{1}{2}\right)\right]$. By comparing the energies we determine the phase diagrams shown in Fig. 1.

In the ferromagnetic-nonmagnetic phase half of the spins are equal to zero and the other half are equal to +1 (or -1). The emergence of this phase can be viewed as a manifestation of the weakening effect of randomness on first-order transitions: the jump, $m= \pm 1$ to $m=0$, at small $H<\frac{1}{4}$ is replaced by two smaller jumps, $m= \pm 1$ to $m= \pm \frac{1}{2}$ and $m= \pm \frac{1}{2}$ to $m=0$, at $\frac{1}{4}<H<\frac{1}{2}$. For even stronger randomness $H>\frac{1}{2}$ the ferromagnetic phase is replaced by the paramagnetic phase. A reentrance phenomenon, or nonmonotonic dependence of densities, takes place in this regime: lowering $D$ the magnetization is first 0 , then $\pm \frac{1}{2}$, then 0 again.
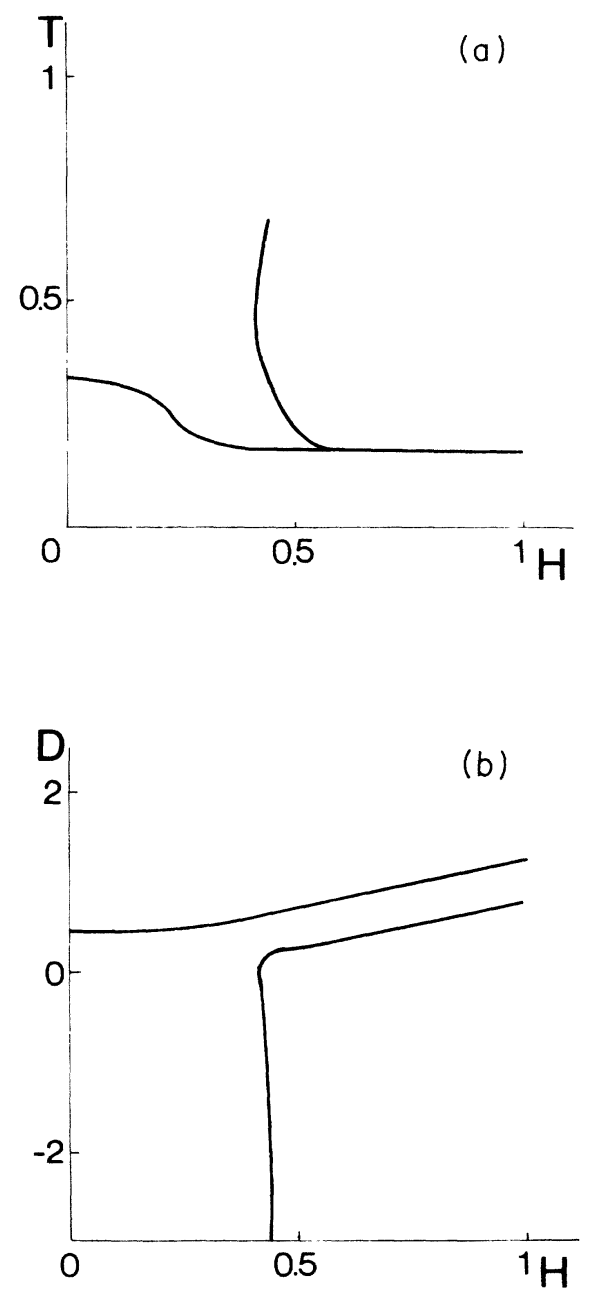

FIG. 2. Projections of the two tricritical lines along the $D$ axis in (a) and along the $T$ axis in $(b)$. 


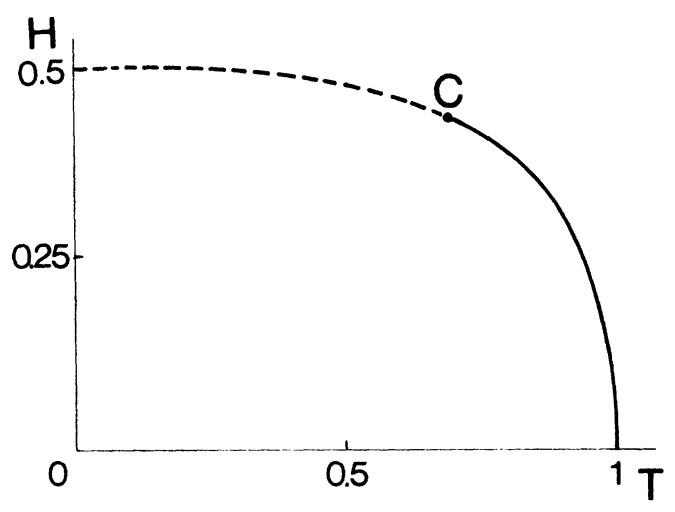

FIG. 3. Phase diagram for $D=-5$ with a tricritical point $C$. The dashed line represents first-order transitions and the solid one is a line of critical points.

\section{B. Tricritical points}

We list the entities on the phase diagram by using Griffiths' notation system: ${ }^{12} \mathrm{~A}$-one-phase point (disordered phase, $m=0$ ), $A^{2}$-two-phase point (ordered phase, $\left\langle s_{i}\right\rangle= \pm|m|$ ), $A^{3}$-three-phase point (coexistence of ordered and disordered phases), $A^{4}$-four-phase point (coexistence of two ordered phases), $A^{5}$-five-phase point (coexistence of two ordered phases and the disordered phase), $B$-critical point, $B^{2}$-ordered-critical point (coexistence of two critical phases), $C$-tricritical points, $B^{2} A$-ordered-critical end point (coexistence of two critical phases and the disordered phase).

The location of the tricritical points is determined by expanding $\Phi(m)$ in powers of $m$ :

$$
\Phi(m)-\Phi(0)=a_{2} m^{2}+a_{4} m^{4}+a_{6} m^{6} .
$$

The fields $a_{2}, a_{4}$, and $a_{6}$ depend on $T, H$, and $D$ according to

$$
\begin{aligned}
a_{2}= & \frac{J^{2}}{2 !}\left[\frac{1}{J}+w^{2} u-w\right], \\
a_{4}= & \frac{J^{4}}{4 !}\left[6 w^{6} u^{2}-12 w^{3} u+w^{2}(4 u+3)-w\right], \\
a_{6}= & \frac{J^{6}}{6 !}\left[120 w^{6} u^{3}-360 w^{5} u^{2}+w^{4}\left(120 u^{2}+270 u\right)\right. \\
& \left.\quad-w^{3}(150 u+30)+w^{2}(16 u+15)-w\right],
\end{aligned}
$$

where

$$
\begin{aligned}
& u=\tanh ^{2}(J H), \\
& w=e^{-J D} 2 \cosh (J H)\left[1+e^{-J D} 2 \cosh (J H)\right]^{-1},
\end{aligned}
$$

and $J=1 / T$. The tricritical points $C$ occur at $a_{2}=a_{4}=0$ and $a_{6}>0$. We checked ${ }^{13}$ that $a_{6}>0$ in the entire $T, H, D$ space and thus there is no fourth-order point in this model.

In the $T, H, D$ space the tricritical manifold contains two noncontiguous lines. The first starts at the tricritical point of the pure Blume-Capel model: ${ }^{4} H=0$, $D=(\ln 4) / 3, T=\frac{1}{3}$. The second line starts at the tricriti-

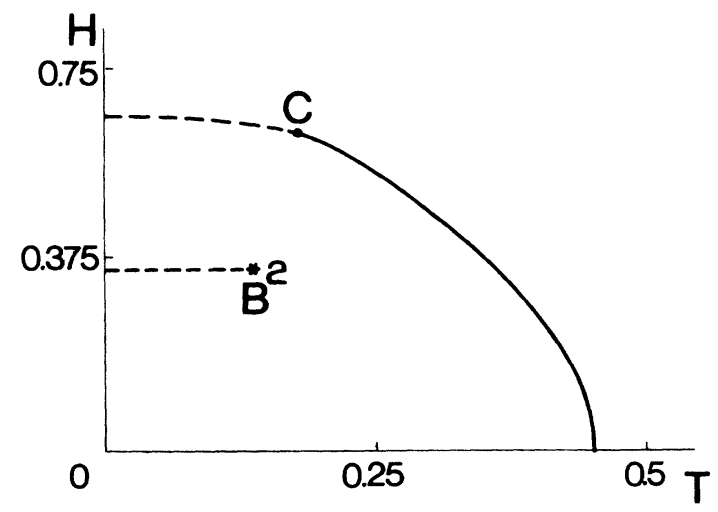

FIG. 4. Phase diagram for $D=0.4$ with a tricritical point $C$ and an ordered critical point $B^{2}$. The dashed lines represent first-order transitions and the solid one is a line of critical points.

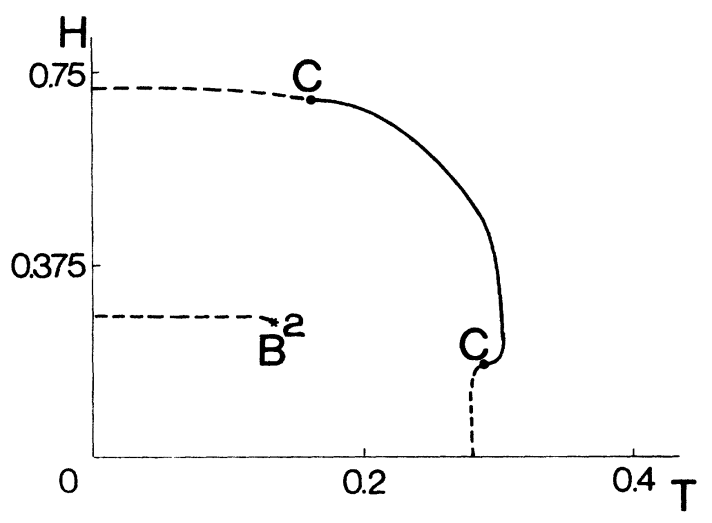

FIG. 5. Phase diagram for $D=0.47$ with two tricritical points $-C$ and an ordered critical point $B^{2}$. The dashed lines represent first-order transitions and the solid one is a line of critical points.

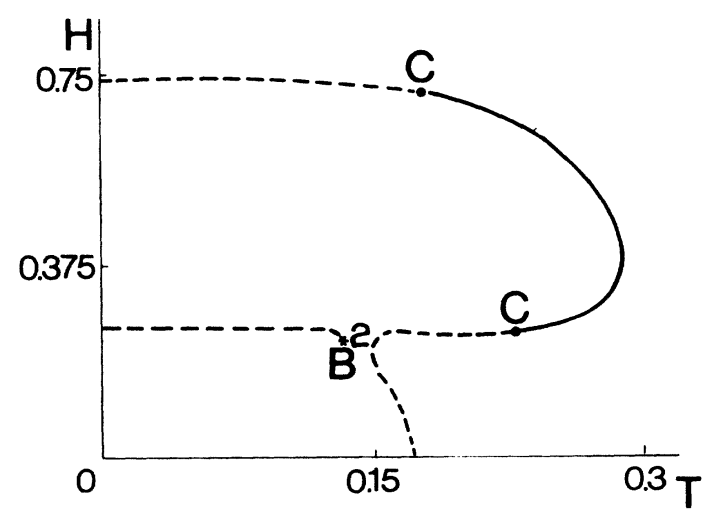

FIG. 6. Phase diagram for $D=0.492$ with two tricritical points $C$ and an ordered critical point $B^{2}$. The dashed lines represent first-order transitions and the solid one is a line of critical points. The gap between $B^{2}$ and the first-order line $A^{3}$ is about to be closed. 


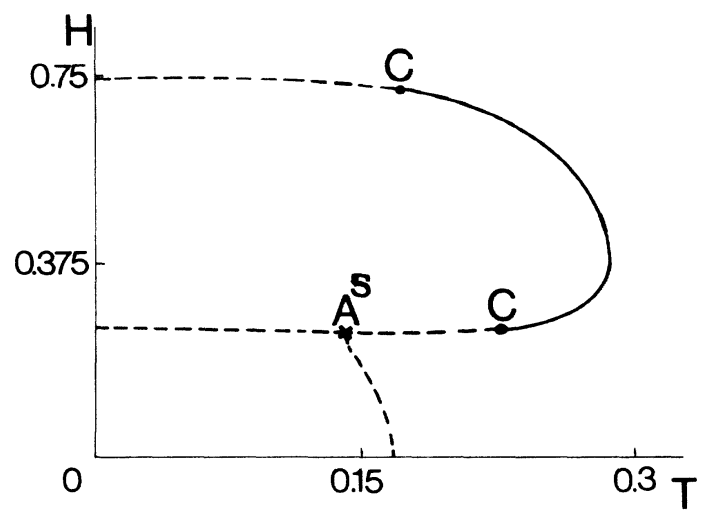

FIG. 7. Phase diagram for $D=0.493$ with two tricritical points $C$ and an $A^{5}$ point. The dashed lines represent first-order transitions and the solid one is a line of critical points.

cal point of the Ising model in a bimodal random field: ${ }^{11}$ $D=-\infty, \quad H=[\ln (2+\sqrt{3})] / 3, \quad T=\frac{2}{3}$. Projections of these lines along $D$ and $T$ directions are shown in Fig. 2. The first tricritical line exhibits the expected monotonic decrease of $T$ with $H$. However the second tricritical line exhibits a monotonic dependence of $T$ and $D$ on $H$. It has an extremum at $T \cong 0.46, H \cong 0.41, D \cong 0$, corresponding to a double tricritical point (see Appendix A of Ref. 7).

\section{Global phase diagram}

We determine the phase diagram by numerically minimizing $\Phi$ given in Eq. (4). We choose to present two-dimensional slices, at fixed $D$, of the threedimensional parameter space. There are five topologically different such two-dimensional phase diagrams.

For $D<\frac{1}{4}$ the phase diagram, Fig. 3, is topologically equivalent to Aharony's phase diagram ${ }^{11}$ for the Ising model in a bimodal random field, which includes a tricritical point.

For $\frac{1}{4} \leq D<(\ln 4) / 3$ there is a line of $A^{4}$ points ending

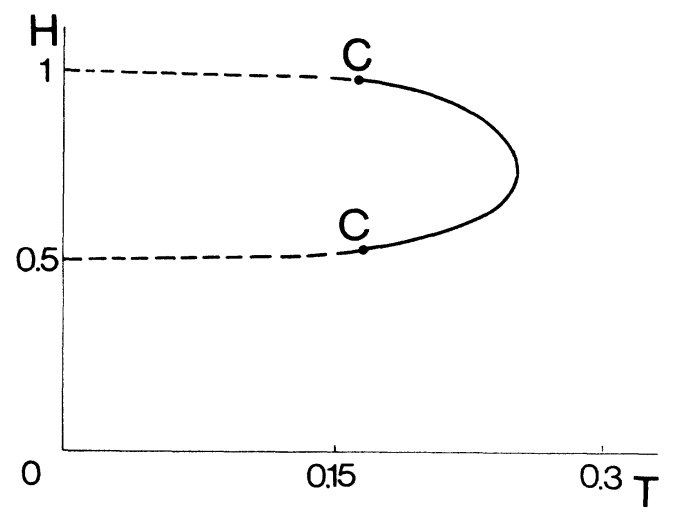

FIG. 8. Phase diagram for $D=0.75$ with two tricritical points $C$. The dashed lines represent first-order transitions and the solid one is a line of critical points. at a $B^{2}$ point inside the ordered phase (see Fig. 4). Along this line the ferromagnetic and ferro-nonmagnetic phases coexist. At the ordered critical point the two phases coalesce.

For $D=(\ln 4) / 3$ a second tricritical point emerges as $H=0, T=\frac{1}{3}$. Thus for $(\ln 4) / 3 \leq D<0.493$ there are two tricritical $C$ points and one ordered critical $B^{2}$ point, see Figs. 5 and 6 .

At $D \cong 0.493$ (slightly below this value), $T \cong 0.14$, $H \cong 0.24$, there is an ordered-critical end point $B^{2} A$, which viewed in the three-dimensional parameter space is located at the intersection of the $B^{2}$ line and the $A^{3}$ surface. To our knowledge this multicritical point has not been observed in any previous theoretical or experimental study. For $0.493 \leq D<\frac{1}{2}$ this multicritical point is replaced by an $A^{5}$ point, see Fig. 7. As $D$ approaches $\frac{1}{2}$, the $A^{5}$ point and the segment of $A^{3}$ points below it approach the $T=0$ axis.

For $D \geq \frac{1}{2}$ the ferromagnetic-nonmagnetic phase is separated from the disordered phase $(m=0)$ by a phase boundary consisting of two first-order transitions segments and a segment of critical points. There are two tricritical points in this case, see Fig. 8.

\section{CONCLUSIONS AND DISCUSSION}

We have determined the global phase diagram of the Blume-Capel model in a bimodally distributed random field by using the the mean-field approximation. The highest-order multicritical entity is the tricritical point. An ordered critical end point is also included in the phase diagram

A novel phase, the ferromagnetic-nonmagnetic phase emerges at sufficiently strong random fields. We believe that this phase will also occur for realistic short-range interactions in three dimensions. Indeed at low temperatures and for large $H$ the magnetic spins are favored over the nonmagnetic ones and simultaneously are randomized by the random field: $m=0, Q=1$. At large $D$, on the other hand, the nonmagnetic spins are favored: $m=0, Q=0$. What happens in the intermediate region $H \approx D$ ? We envision three scenarios: (i) the two phases are separated by a special first-order transition where $Q$ jumps from 1 to 0 but $m$ stays equal to 0 ; this dichotomy in the behavior of the two densities makes this scenario less likely; (ii) there is a continuous transition; $Q$ varies from 1 to 0 in the paramagnetic phase and $m$ stays equal to 0 ; this scenario could work in two dimensions where randomness causes first-order transitions to be replaced by continuous transitions; ${ }^{1,2}$ (iii) the ferromagneticnonmagnetic phase emerges as a buffer between the paramagnetic and the nonmagnetic phases and is separated from them by first-order transitions; this is the meanfield behavior and we expect it holds in three dimensions. It will be very interesting to check whether this phase indeed emerges in three dimensions by using an alternative technique (Monte Carlo, renormalization group).

The effect of randomness on the tricritical points is more complicated than anticipated. There are two noncontiguous tricritical lines. On one of them the tricritical temperature decreases monotonically with the strength of 
the random field, as expected. On the second line however, there is a double tricritical point located at an extremum on the tricritical line, i.e., nonmonotonic dependence.

\section{ACKNOWLEDGMENTS}

We are grateful to P. E. Klunzinger for help with the computer programming.
${ }^{1}$ K. Hui and A. N. Berker, Phys. Rev. Lett. 62, 2507 (1989).

${ }^{2}$ M. Aizenman and J. Wehr, Phys. Rev. Lett. 62, 2503 (1989).

${ }^{3}$ T. Schneider and E. Pytte, Phys. Rev. B 15, 1519 (1977).

${ }^{4}$ M. Blume, Phys. Rev. 141, 517 (1966); H. W. Capel, Physica 32, 966 (1966).

${ }^{5}$ M. Blume, V. J. Emery, and R. B. Griffiths, Phys. Rev. A 4, 1071 (1971).

${ }^{6}$ A. N. Berker and M. Wortis, Phys. Rev. B 14, 4946 (1976).

${ }^{7}$ M. Kaufman, R. B. Griffiths, J. M. Yeomans, and M. E. Fisher, Phys. Rev. B 23, 3448 (1981).

${ }^{8}$ For a review of tricritical models, see I. D. Lawrie and S. Sarbach, in Phase Transitions and Critical Phenomena, edited by C. Domb and J. L. Lebowitz (Academic, New York, 1984), Vol. 9, p. 1.
${ }^{9}$ Transitions in which only one density undergoes a jump while other densities vary continuously are rare but not unheard of, see the following: D. J. Thouless, Phys. Rev. 187, 732 (1969); M. Kaufman and R. B. Griffiths, Phys. Rev. B 26, 5282 (1982).

${ }^{10}$ M. Kaufman, P. E. Klunzinger, and A. Khurana, Phys. Rev. B 34, 4766 (1986).

${ }^{11}$ A. Aharony, Phys. Rev. B 18, 3318 (1978).

${ }^{12}$ R. B. Griffiths, Phys. Rev. 12, 345 (1975).

${ }^{13}$ The stability condition $a_{6}>0$ was not checked in R. M. Sebastianes and V. K. Saxena, Phys. Rev. B 35, 2058 (1987) thus resulting in a partially erroneous phase diagram for the Ising model in a trimodal random field. The correct phase diagram of this model was first presented in Ref. 10. 